\title{
External Validation of the WILL-BLEED Risk Score
}

Fausto Biancari, ${ }^{1,2,3}$ Tuomas Tauriainen, ${ }^{2}$ Eeva-Maija Kinnunen, ${ }^{2}$ Juhani Airaksinen $^{3}$

${ }^{1}$ Department of Surgery, University of Turku, Turku, Finland; ${ }^{2}$ Department of Surgery, University of Oulu, Oulu, Finland;

${ }^{3}$ Heart Center, Turku University Hospital, Turku, Finland.

Running title: Will-Bleed Score

Keywords: Coronary artery bypass; bleeding; anemia; transfusion.

Grant support: None

Conflicts of interest: None to declare

Manuscript word count: 672 words

For correspondence:

Prof. Fausto Biancari,

Heart Center,

Turku University Hospital,

Hämeentie 11, PL 52, 20521 Turku, Finland

Tel.: +358407333973

E-mail: faustobiancari@yahoo.it 
To the Editor:

Perioperative bleeding is recognized as a major complication affecting the early and late outcome of patients undergoing cardiac surgery (1). Such a risk is of particular relevance in patients undergoing coronary artery bypass grafting (CABG) because they are often exposed preoperatively to potent antithrombotics and not infrequently require urgent or emergency operation, which prevents the discontinuation of these drugs. Recently, we developed a risk scoring method, the WILL-BLEED score, for prediction of severe and massive perioperative bleeding in patients undergoing CABG (2). The value of this risk score was questioned because of the lack of an external validation $(1,3)$. Therefore, we sought to validate its predictive ability in an institutional series.

The present study includes 2761 patients who underwent isolated CABG from June 2006 to December 2013 at the Oulu University Hospital, Finland. Data on pre-, intra- and postoperative variables were retrospectively collected. Data on the types and amount of blood products transfused in these patients were retrieved from a prospective electronic hospital registry. Preoperative anemia was defined a hemoglobin level $<12.0 \mathrm{~g} / \mathrm{dL}$ in women and $<13.0 \mathrm{~g} / \mathrm{dL}$ in men. The outcome measures of this analysis were the amount of units of transfused red blood cells (RBC), transfusion of $>4$ units of RBC and/or resternotomy for bleeding, and 30-day mortality.

Statistical analysis was performed using an SPSS statistical software (version 24.0 IBM Corporation; Armonk, New York, USA). Continuous variables were reported as the mean and standard deviation as well as median and interquartile range where appropriate. Nominal variables were reported as counts and percentages. Pearson Chi-square and Mann-Whitney tests were used for univariate analysis. The Spearman's test was used to evaluate the correlation between the WILL-BLEED risk score and the amount of transfused units of RBC. 
Discriminatory ability and calibration of the WILL-BLEED risk score in predicting the study outcomes were assessed by evaluating the area under (AUC) the receiver operating characteristic (ROC) curve and by the Hosmer-Lemeshow (H-L) test, respectively. The WILLBLEED risk score was also dichotomized according to the previously identified cut-off value of 5. All tests were two-sided with the alpha level set at 0.05 for statistical significance.

Details on this series are reported elsewhere (4). In brief, the mean age of these patients was

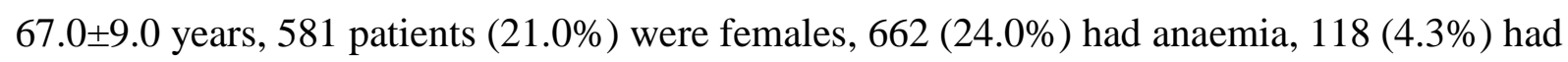
an estimated glomerular filtration rate $<45 \mathrm{ml} / \mathrm{min} / 1.73 \mathrm{~m}^{2}, 1611(58.3 \%)$ had acute coronary syndrome, $1502(54.4 \%)$ required urgent or emergency operation and 217 (7.9) were in critical preoperative status. Thirty-day mortality rate was $3.2 \%$. RBC transfusion was required in $63.5 \%$ of patients and resternotomy for bleeding in $6.5 \%$ of patients. Transfusion of $>4$ units of RBC was observed in $18.5 \%$ of patients and transfusion of $>4$ units of RBC and/or resternotomy for bleeding was necessary in $20.7 \%$ of patients and increased along with the WILL-BLEED risk score ( $\mathrm{p}<0.0001$, Fig. 1).

The WILL-BLEED risk score significantly correlated with the amount of transfused RBC (rho: $0.486, \mathrm{p}<0.0001$ ). The WILL-BLEED risk score showed good discriminatory ability, but suboptimal calibration for prediction of transfusion of $>4$ units of RBC and/or resternotomy for bleeding (AUC of ROC, 0.721, 95\%CI 0.697-0.745, H-L test: $\mathrm{p}=0.082$ ), transfusion of $>4$ units of RBC (AUC of ROC, 0.756, 95\%CI 0.733-0.780, H-L test: $\mathrm{p}=0.016$ ) and 30-day mortality (AUC of ROC, 0.754, 95\%CI 0.697-0.811, H-L test: $\mathrm{p}=0.106$ ).

A WILL-BLEED risk score $>5$ was associated with a significantly increased amount of transfused RBC units (median $4.0 \pm 4.0$ vs. $1.0 \pm 3.0$ units, mean $4.7 \pm 4.5$ vs. $1.8 \pm 2.5$ units, $\mathrm{p}<0.0001)$ and of risk of transfusion of $>4$ units of RBC and/or resternotomy for bleeding (42.8\% vs. $13.5 \%, \mathrm{p}<0.0001)$ and 30 -day mortality $(8.7 \%$ vs. $1.4 \%, \mathrm{p}<0.0001)$. 
This analysis showed that the WILL-BLEED risk score correlated with the amount of transfused RBC and has a good discriminatory ability of predicting transfusion of $>4$ units of $\mathrm{RBC}$ and/or resternotomy for bleeding and 30-day mortality. This external validation study showed that the calibration of this score is suboptimal as shown in the derivation dataset of this score as well as of other bleeding risk scores (2).

This external validation analysis showed that the WILL-BLEED risk score could be a valuable means to stratify the risk of perioperative bleeding in patients undergoing isolated CABG. The predictive ability of this bleeding risk score should be evaluated in patients undergoing heart valve surgery and in those undergoing percutaneous coronary intervention.

\section{References}

1. Pagano D, Milojevic M, Meesters MI, et al. 2017 EACTS/EACTA Guidelines on patient blood management for adult cardiac surgery: The Task Force on Patient Blood Management for Adult Cardiac Surgery of the European Association for CardioThoracic Surgery (EACTS) and the European Association of Cardiothoracic Anaesthesiology (EACTA). J Cardiothorac Vasc Anesth 2017 (in press)

2. Biancari F, Brascia D, Onorati F, et al. Prediction of severe bleeding after coronary surgery: the WILL-BLEED Risk Score. Thromb Haemost 2017;117:445-456.

3. Roldán V, Marín F. Predicting bleeding risk after coronary surgery: Let's focus on modifiable risk factors and simple, practical decision making. Thromb Haemost 2017;117:647-649.

4. Tauriainen T, Koski-Vähälä J, Kinnunen EM, et al. The effect of preoperative anemia on the outcome after coronary surgery. World J Surg 2017;41:1910-1918. 
- $\mathrm{RBC}$ transfusion $>4$ units

a $\mathrm{RBC}$ transfusion $>4$ units and/or resternotomy

51.151 .5

50

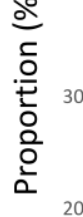

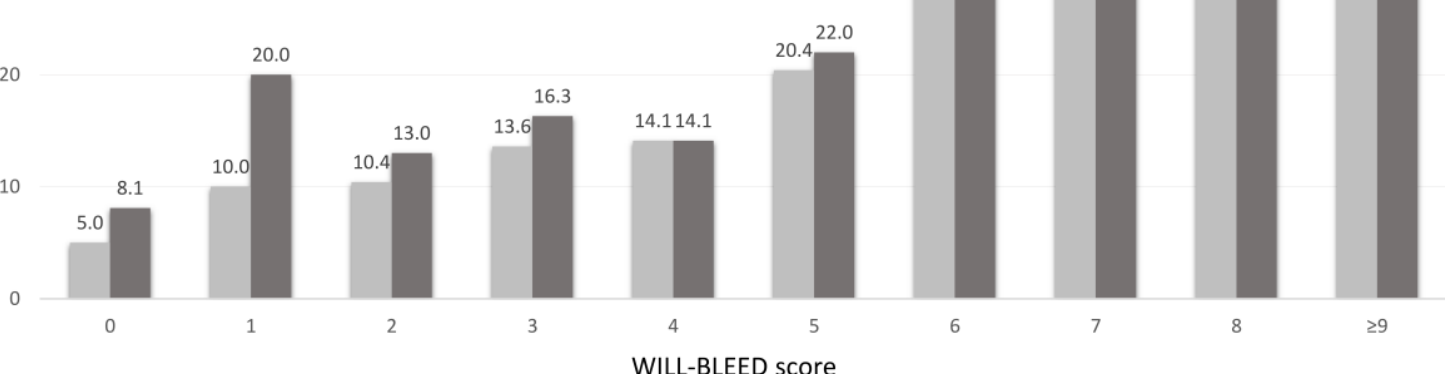

\section{Legend to figure}

Figure 1. Proportion of transfusion of $>4$ units of red blood cells (RBC) and/or resternotomy for bleeding after coronary artery bypass grafting according to the WILL-BLEED risk score. 